\title{
Allgemeinbildung in Zeiten der Globalisierung
}

\author{
Meinert A. Meyer • Annette Scheunpflug $\cdot$ Stephanie Hellekamps
}

(C) Springer Fachmedien Wiesbaden GmbH, ein Teil von Springer Nature 2018

Eine der zentralen Annahmen im Wissenschaftsdiskurs ist jene, dass angesichts der Globalisierung sich die Diskurse internationalisieren bzw. auf einander Bezug nehmen. In diesem Heft möchten wir einen Beitrag zur Wahrnehmung des Diskurses um „Allgemeinbildung“ in Zeiten der Globalisierung leisten. Überraschend war es nun während der Arbeit an diesem Heft festzustellen, dass sich gerade im Hinblick auf das Thema „Allgemeinbildung“ der gegenseitige Bezug und die gegenseitige Wahrnehmung nicht nur nicht zeigen, sondern darüber hinaus auch die konzeptuellen Vorstellungen dessen, was Allgemeinbildung angesichts der Globalisierung ausmachen könnte, weit auseinandergehen. Es gibt offensichtlich kaum Überlappungen zwischen den einzelnen Entwürfen und die gegenseitige Wahrnehmung ist gering. Wir arbeiten, was das Verständnis von Allgemeinbildung und Globalisierung angeht, offensichtlich immer noch in national und kulturell getrennten Welten. Dass dem tatsächlich so ist, kann der Leser schon durch einen Blick in die Literaturlisten der in diesem Heft versammelten Beiträge feststellen. Aber auch ein Blick in die deutschsprachige Allgemeine Didaktik verweist auf die Problemlast: Wir können

Prof. i.R. Dr. M. A. Meyer

Schulpädagogik und Allgemeine Didaktik, Fakultät für Erziehungswissenschaft, Universität

Hamburg, Hamburg, Deutschland

E-Mail: meinert.meyer@uni-hamburg.de

Althausweg 139, 48159 Münster, Deutschland

Prof. Dr. A. Scheunpflug $(\bowtie)$

Lehrstuhl für Allgemeine Pädagogik, Otto-Friedrich-Universität Bamberg,

Markusplatz 3, 96047 Bamberg, Deutschland

E-Mail: annette.scheunpflug@uni-bamberg.de

Prof. Dr. S. Hellekamps

Historische Schul- und Curriculumforschung, Institut für Erziehungswissenschaft, Westfälische Wilhelms-Universität Münster, Bispinghof 5/8, 48143 Münster, Deutschland

E-Mail: hellekam@uni-muenster.de 
zum Beispiel auf Wolfgang Klafki hinweisen. Er beansprucht, dass sein Modell einer kritisch-konstruktiven Didaktik im Prinzip den Unterricht in kulturell differenten Kontexten strukturieren könne (Klafki 1998, S. 248 f.), bezieht sich gleichwohl aber nicht auf nicht-deutsche didaktische Vorstellungen oder Modelle.

Der große Variantenreichtum didaktischer Konzepte zur Allgemeinbildung wird, so vermuten wir, dadurch gefördert, dass auch auf politischer Ebene die Reform der Schulsysteme und die Verbesserung der Unterrichtsqualität eher als nationale Aufgaben und weniger im globalisierten Kontext wahrgenommen werden. Gegenläufig ist jedoch festzustellen, dass es in Europa ein zunehmendes Interesse an der Klärung der Frage gibt, ob sich Bildung als schulpädagogisch-didaktische Aufgabe in die nationalen didaktischen Konzeptionen für Schule und Unterricht integrieren lässt und ob dadurch ein Forschungsstand „beyond fragmentation“ erreicht werden kann (vgl. Hudson und Meyer 2012). Exemplarisch verweisen wir im Hinblick auf die Artenvielfalt in der Didaktik auf die in Frankreich entwickelte und von John Dewey's „Democracy and Education“ inspirierte Théorie de l'action conjointe en didactique (vgl. Sensevy 2011), die in Deutschland praktisch nicht zur Kenntnis genommen wird. Ein Reflexions-Statement bezüglich der Beiträge zu diesem Themenheft und anderer didaktischer Arbeiten kann darin gesehen werden, dass es sich bei den verschiedenen didaktischen Modellen einschließlich ihrer Allgemeinbildungskonzeptionen um didaktische Konstruktionen handelt, eingebettet in allumfassende Sinnkonstruktionen (so z.B. auch jüngst Rucker 2017).

Wir verdeutlichen unsere Problemlage für dieses Themenheft an knappen Reflexionen zu den sechs Beiträgen dieses Themenheftes (sechs, weil wir Johannes Drerups Sammelrezension einbinden). Dabei wird eine These von Ulrich Beck mit Leben gefüllt, die den Globalisierungsprozess als einen Prozess zunehmender Pluralisierung begreift (Beck 2015 [1997], S. 27; vgl. den Beitrag von Roselius und Meyer in diesem Heft).

Auf den ersten Blick hat man den Eindruck, dass Katharina Roselius und Meinert A. Meyer ausgerechnet mit ihrem das Heft einleitenden Stichwortartikel das Thema verfehlt haben. Sie entwickeln ein Konzept der Globalisierung und der Bildung (nicht der Allgemeinbildung), und sie bewegen sich dabei durchgehend auf dem Niveau der Konzeptbildung, nicht auf einem Niveau, auf dem man fragen kann, wie denn nun konkret in den Schulen Bildung gefördert wird und wie es damit in der sich globalisierenden Welt weitergehen soll. Dabei versuchen sie, nicht-normativ zu argumentieren, obwohl die Geschichte der Didaktik seit Johann Amos Comenius (1570-1670) Allgemeinbildung normativ entfaltet hat. Mit zweitem Blick wird dann aber deutlich, dass das, was zunächst wie Themenverfehlung aussah, indirekt auf offene Fragen in den folgenden Beiträgern des Themenheftes hinweist. Denn wie soll man substanziell über Allgemeinbildung nachdenken, wenn man nicht zugleich über Bildung nachdenkt? Und wie soll man „echo chambers“, d.h. auf sich selbst fixierte Lebenswelten, im Forschungsprozess vermeiden, wenn man sich selbst als Forschende aus der Gefährdungszone herausdiskutiert?

Leif Moos und Theo Wubbels artikulieren am deutlichsten die demokratische Perspektive der abendländischen Bildungstradition, die sie - ähnlich wie Zhengmei Peng und Juan Gu auf der einen Seite und Herwig Blankertz und andere auf der anderen Seite - am Emanzipationsbegriff festmachen. Sie verbinden diesen Ansatz 
dann konsequent mit einer Kritik an Bildungskonzeptionen, die aus dem Einfluss der Ökonomie auf die Gestaltung von Schule und Unterricht eine direkte Abhängigkeit akzeptieren wollen. Angesichts des dramatischen transnationalen Wandels unserer Lebenswelten sehen sie die Notwendigkeit, gegen Uniformierung im Bildungswesen anzugehen. Sie wehren sich gegen ,homogenisierte“ Allgemeinbildung für die globalisierte Welt, was indirekt auch in dieser Perspektive die Pluralitätsthese Ulrich Becks stärkt.

Zhengmei Peng und Juan Gu (unter Mitarbeit von Meinert A. Meyer) sehen sich und sehen wir vor einer beachtlichen Herausforderung. Wie können sie ihr Konzept einer konfuzianischen Allgemeinbildung gegenüber den Allgemeinbildungskonstruktionen der westlichen Welt verteidigen? Sie lösen das Problem durch die Darstellung der mehr als 2000-jährigen konfuzianischen Bildungstradition, die sie dann benutzen, um einen (jedenfalls aus ihrer Sicht) Ost-West-Kompromiss zu finden. Dies wird im Titel des Beitrags schon angedeutet. Die beiden Shanghaier Autoren wollen die Idee einer emanzipatorischen Harmonie konkretisieren, wobei man wissen muss, dass „Harmonie“ der Schlüsselbegriff der konfuzianischen Bildungstradition ist. Es ist deshalb sinnvoll, den Begriff der Emanzipation als Schlüsselbegriff der westlichen Welt (vgl. als Beleg Blankertz 1982, S. 307) dem der Harmonie gegenüber zu stellen. Dabei hat das Konzept der Emanzipation auch in China zu Beginn des 20. Jahrhunderts - eine bedeutsame Rolle gespielt, ist dann aber in kommunistischer Zeit durch die Kulturrevolution überrollt worden.

$\mathrm{Ob}$ es heute, trotz lebhafter Diskussionen unter chinesischen Didaktikern, eine neue kompromissorientierte Allgemeinbildung geben kann, ist - so Peng und Gu noch nicht geklärt. Diese neue Allgemeinbildung müsste Peng u.a. zufolge, wenn sie Emanzipation als didaktische Sinnkonstruktion in ihr Modell integrieren soll, zugleich die Dominanz der Harmonie als oberster Sinnkonstruktion akzeptieren. Peng und Gu sind in dieser Hinsicht optimistisch: „Man kann [...] von einer emanzipatorischen Wende der konfuzianischen Allgemeinbildung sprechen, wobei aber die harmonische Tradition nicht ihren Vorrang verliert". Dieser für eine abendländische Theorie- und Argumentationsstruktur etwas ungewöhnliche Beitrag entwickelt vor dem Hintergrund einer chinesisch-geschlossenen Theorieargumentation des Konfuzianismus ein Verständnis Allgemeiner Didaktik, das sich nur an wenigen Stellen - und dann meist abgrenzend - dem internationalen Diskurs gestellt hat. Mit der Veröffentlichung dieses Aufsatzes möchte die ZfE einen Beitrag zu einem Dialog mit dieser in der amerikanisch-europäisch Welt eher selten rezipierten Form didaktischen Denken leisten.

Bernard Schneuwly kann zu Recht monieren, dass die frankophone didaktische Tradition bis jetzt in Deutschland kaum zur Kenntnis genommen worden ist. Er schafft es dennoch, in einem historischen Rückblick auf Wilhelm von Humboldt und den Marquis de Condorcet, die preußisch-deutsche und die frankophone Tradition zusammenzubringen und so zugleich ihre Verschiedenheit herauszustellen. Humboldt fordert, ähnlich wie Konfuzius, für den Lernprozess zum einen „Mannigfaltigkeit der Situationen“, in welchen Schüler lernen können, und zum anderen „Freiheit“, um in diesen Situationen eigenständig zu lernen. Auf dieser Basis zeigt Schneuwly, dass Schulfächer als soziohistorische „Form“ des Schulsystems verstanden werden können und sich in dieser „Form“ das, was wir objektiv, als 
vorzugebenden Lerninhalt, unter Bildung verstehen, ständig weiterentwickelt. Der in der Frankophonie zentrale Begriff für die Organisation des Curriculums ist dabei der der „discipline“ in seiner Doppelbedeutung. Er bringt Ordnung in das Ensemble der Unterrichtsfächer, aber er diszipliniert zugleich die Akteure, Schüler wie Lehrer. Die Schulfächer sind dabei „Kampfplätze“ der gesellschaftlichen Entwicklung, was Erich Weniger ähnlich gesehen hatte.

Christine Sälzer und Nina Roczen behandeln ein spannendes Thema, das es normalerweise nicht bis zur Publikation schafft - den partiellen Rückzug aus einem Forschungsvorhaben, und nicht aus irgendeinem, sondern aus dem Bereich ,globale Kompetenz“ von PISA 2018. Das DIPF in Frankfurt als verantwortlicher deutscher Kooperationspartner zieht sich partiell zurück, weil das Konstrukt der ,global competence" der 15-Jährigen aus deutscher Sicht noch nicht so weit entwickelt ist, wie es sein müsste, um damit in die Hauptphase von PISA $2018 \mathrm{zu}$ gehen. Zu fragen ist deshalb, ob das ,noch nicht“ auf noch nicht erledigte Arbeit verweist oder ob es prinzipielle Grenzen für ein derartiges Projekt gibt, das sowohl hinsichtlich der Aufgabenstellung globaler Kompetenzen selbst wie auch deren kulturübergreifender Messbarkeit universale Ansprüche erhebt. Der Beitrag von Sälzer und Roczen könnte als Exempel dafür dienen, dass der Globalisierungsprozess ein Pluralisierungsprozess ist. Während die beiden Autorinnen offensichtlich davon ausgehen, dass die Operationalisierung des Konzepts im Prinzip möglich sein muss, stärken unsere Erkenntnisse im Hinblick auf die Diversität, wie wir sie in diesem Themenheft gebündelt haben, eher die Beck'sche These der zunehmenden Pluralisierung.

Auch Johannes Drerup liefert mit seiner Sammelrezension einen markanten Beitrag zu unserem Thema. Er kann zeigen, dass in ausgewiesenen Bereichen - sein Beispiel ist die Global Citizen Education - durchaus schon erfolgreich über die Globalisierung der Curricula nachgedacht wird, und er liefert dazu auch gleich eine allgemein bekannte einrahmende Sinnkonstruktion: „Think global, act local!“ Drerup thematisiert damit das Programm der Kanonbildung und implizit das Programm der Erstellung eines Weltallgemeinbildungscurriculums. Dabei kann es aus unserer Sicht nicht darum gehen, im Stile eines geschlossenen Lehrplanwerks die Unterrichtsinhalte vorherbestimmen zu wollen. Aber wie man Globalisierung und lokale wie regionale Individualität zusammendenken soll, ist eine offene Frage. Auf der Basis der Erkenntnisse, wie sie in diesem Themenheft vorgestellt werden, würden wir ein solches Unternehmen skeptisch beurteilen.

Insgesamt wird deutlich: Offensichtlich befinden sich die deutsche und die internationale Didaktik bezüglich der Frage eines gemeinsam wahrgenommenen Diskursfeldes noch in den Anfängen. Und wenn dem so ist, dann hat das Auswirkungen auf die bestmögliche Gestaltung der didaktischen Forschung. Qualitative, Forschungsfelder erkundende Verfahren sind dann neben der quantitativen empirischen Bildungsforschung angesagt. Und dies gilt umso eher, als manche Politiker, Lehrende und Erziehungswissenschaftler Globalisierung als Expansion der Nationalstaaten denken. Sie denken Globalisierung also nicht als transnationalen und transformatorischen Prozess, der mehrperspektivisches Denken erfordert.

Die Explikation der nationalen Selbstverständlichkeiten und Verschiedenheiten, die sich ergeben, wenn man die Globalisierung der Lebensbedingungen hinzunimmt, ist offensichtlich weiteres Nachdenken wert. 
In der Zusammenschau der Beiträge dieses Hefts zeigt sich die Forschungsherausforderung. Mit den jeweils genannten Autoren können die folgenden Fragen gestellt werden:

- Wie lässt sich das Konzept der Allgemeinbildung im Kontext von Globalisierung kulturell nicht-normativ beschreiben und soll es überhaupt beschrieben werden? (Roselius/Meyer)

- Gibt es einen Konsens dahingehend ob und in welcher Form Allgemeinbildung zur Förderung von Demokratie beitragen können soll und wie lässt sich dieser Konsens ggf. fassen? (Moos/Wubbels) Oder spielen andere Kategorien, wie etwa das chinesische Konzept der „emanzipativen Harmonie“ eine Rolle? (Peng/Gu/ Meyer)

- Welche Rolle kann das Konzept einer wie auch immer gefassten Allgemeinbildung im Kontext der Steuerung von Bildungssystemen angesichts der Globalisierung spielen?

- Welche Rolle spielt die Formation der Schule in Fachstrukturen und angesichts der Ansprüche an eine Globalisierung des Wissens? (Schneuwly) Wird in diesem Kontext ein minimaler Konsens über ein Weltallgemeinbildungscurriculum benötigt? (Drerup)

- Wie lassen sich Allgemeinbildungskonzepte kulturübergreifend so operationalisieren, dass sie für eine Testung vergleichbar werden? Oder sind die doppelten Ansprüche an kulturelle Homogenisierungen im Gegenstandsbereich und in der Testmethode so groß, dass der damit zu beschreibende Bildungszuwachs diffundiert? (Sälzer/Roczen)

Obwohl die Globalisierung der Lebenswelt eines der ganz großen Zukunftsthemen darstellt, steckt die Klärung der Frage, was dies für Bildung und Allgemeinbildung bedeutet, immer noch in den Anfängen. Das vorliegende Heft soll zu diesem unterentwickelten Forschungsfeld einen Beitrag leisten, indem verschiedene nationale Diskurskontexte aufeinander bezogen werden.

\section{Literatur}

Beck, U. (2015). Was ist Globalisierung? Irrtümer des Globalismus - Antworten auf Globalisierung (3. Aufl.). Frankfurt a. M.: Suhrkamp.

Blankertz, H. (1982). Die Geschichte der Pädagogik. Von der Aufklärung bis zur Gegenwart. Wetzlar: Büchse der Pandora.

Hudson, B., \& Meyer, M.A. (Hrsg.) (2012). Beyond fragmentation: didactics, learning and teaching in europe. Opladen: Barbara Budrich.

Klafki, W. (1998). Schlüsselprobleme der modernen Welt und die Aufgaben der Schule - Grundlinien einer neuen Allgemeinbildungskonzeption in internationaler/interkultureller Perspektive. In I. Gogolin, M. Krüger-Potratz, \& M. A. Meyer (Hrsg.), Pluralität und Bildung (S. 235-249). Opladen: Leske + Budrich.

Rucker, T. (2017). Allgemeine Didaktik als Reflexionsinstanz. Zeitschrift für Pädagogik, 63(5), 618-635.

Sensevy, G. (2011). Le sens du savoir. Eléments pour une théorie de l'action conjointe en didactique. Brüssel: Groupe De Boeck. 